\title{
Ethics in Adult Orthodontics
}

\author{
Kaushik P. ${ }^{1}$, Lokamithra R. ${ }^{2}$, Arani Nandakumar ${ }^{3}$ \\ ${ }^{1}$ Assistant Professor, Department of Orthodontics and Dentofacial Orthopaedics, Meenakshi Ammal Dental \\ College and Hospital, Meenakshi Academy of Higher Education and Research, Chennai, ${ }^{2}$ Post Graduate student, \\ Department of Orthodontics and Dentofacial Orthopaedics, Meenakshi Ammal Dental College and Hospital, \\ Meenakshi Academy of Higher Education and Research, Chennai, ${ }^{3}$ Professor, Department of Orthodontics and \\ Dentofacial Orthopaedics, Meenakshi Ammal Dental College and Hospital, Meenakshi Academy of Higher \\ Education and Research, Chennai
}

\begin{abstract}
Ethics is the understanding and the ability to differentiate right from wrong in human behavior. Dental ethics denotes moral duties and obligations of a dental practitioner towards their patients, fellow colleagues, and the society at large. An ethical practice among dentists ensure good patient care in concurrence with their treatment needs. Ethical principles and codes introduced by regulatory bodies benefit the patient, avoid harm, and respect patient's preferences. Ethical dilemmas are faced by orthodontists during orthodontic interventions, even though important human values may be at stake during the course of treatment. The code of ethics in dentistry warrants an orthodontist to act in the best interest of their patients regardless of their financial status, even putting themselves at jeopardy if need be. This article thus focuses on the morals and ethics that should be abided by an orthodontist in order to deliver the best possible care to their patients.
\end{abstract}

Keywords: Ethics, Orthodontics, Dental practice, Ethical practice, Dentistry.

\section{Introduction}

The etymology of the term "Ethics" is from the Greek word "Ethikos" which means character or conduct. It is defined as the systematic examination of human behavior. Any decision or action taken to resolve a dilemma should be completely thought through before its initiation. ${ }^{[1]}$

Both medical and dental specialties consist of various ethical principles and values which are strictly followed and respected for many years now. Like any other dental

\section{Corresponding Author: \\ Dr. Kaushik P. MDS}

Assistant Professor, Department of Orthodontics and Dentofacial Orthopaedics, Meenakshi Ammal Dental College and Hospital, Meenakshi Academy of Higher Education and Research, Chennai Phone Number: 09840351383 e-mail: drkaushik.ortho@madch.edu.in procedure, orthodontic interventions deal with ethical dilemmas and hence should be subjected to specific ethical inquiries. Although,it is not every day that an orthodontist faces life or death situation in their practice, however, they deal with important human values which are at stake during the course of orthodontic treatment. Some of these include, pain prevention and management, preservation and restoration of oral function for normal speech and mastication, preservation and restoration of patient's physical appearance, and enhancing a sense of control and responsibility over their health. ${ }^{[2]}$ Apart from treating adult patients, orthodontists also manage a large number of pediatric patients and may encounter ethical issues in that regards it is within the purview of the orthodontist to provide the best treatment option for the child, help in surrogate/guardian decision making, and enable access to care.

This review articlethus highlights the ethical dilemmas faced by an orthodontist in their day to day clinical practice and provides an insight into the effective management measures in accordance with the regulations 
Ethical Principles: Ethical problems usually arise when there is an uncertainty or conflicting moral obligations. ${ }^{[3]}$ The orthodontist should be aware of the ethical principles present for clinical decision making in their practice. The aim of these codes is to enhance a sense of ethical responsibility in order to ensure the highest level of professional and ethical consciousness to maintain a standard of decision making and conduct. ${ }^{[4]}$ The major principles include: ${ }^{[5]}$

1. To do no harm (non-maleficence)

2. To do good (beneficence)

3. Respect for persons

4. Justice

5. Veracity or truthfulness

6. Confidentiality

\section{Ethics in Clinical Practice:}

Inform Before Perform: Once the patient has decided to undergo orthodontic treatment, details of the proposed treatment plan from the start to end until retention phase must be explained to them. The information should be conveyed in a lucid easily understandable language without much usage of complicated dental terms. This can be done using hand held models, patient casts or even showing improvement in profile and frontal views after orthodontic treatment using latest digital software. This essential step before the start of active treatment is useful in encouraging and motivating patients towards the treatment, thus gaining their trust towards the orthodontist

Right to choose: Decisions regarding orthodontic treatment are not entirely decided by the orthodontist. they are required to respect the moral and legal authority of the patients and incase of pediatric patients, the parents. ${ }^{[6]}$ Various treatment options, if any, should be informed and clearly explained to the patients prior to the treatment. The health care professionals should respect the patients right and give them the freedom to decide upon their treatment. Patients must be given full authority in the decision making process and must not be forced to make a decision that is favored by the orthodontist.

Benefit/Risk ratio: The treating orthodontist must first understand the treatment needs of the patientin depth to have a clear view of the type of intervention required for them. Any orthodontic treatment which is considered ideal and beneficial for the patient, may have certain risks and limitations associated with it. Alternative treatment plans options with lesser risks, if any, should be put forth for the patients to decide, which will benefit their oral function, appearance, and quality of life, rather than not doing any treatment. Orthodontists have a moral obligation to support patients' interests and to do no harm. ${ }^{[7]}$

Course of treatment duration: Total time taken for completion of orthodontic treatment depends on the severity of malocclusion. ${ }^{[8]}$ Patients should be informed about the treatment duration prior to start of treatment as the time taken for completion of extraction cases is more than non-extraction cases. Estimated treatment time predicted by an orthodontist can be delayed due to secondary factors like irregular or missed appointments and/or broken appliance or debonded brackets. In case of increase in treatment duration due to the secondary factors the orthodontist should differentiate between their responsibility and that of patients, thereby encourage them to be more regular and responsible towards their treatment. ${ }^{[9]}$

Retention Phase: Before removal of orthodontic appliance, the case should be finished in good occlusion without premature occlusal contacts to ensure long term stability. Post active treatment, retainers should be given with instructions on their timely wear to prevent relapse. Importance of retainers in maintaining the corrected malocclusion should be informed prior to the start of treatment and should be emphasized on the duration of wear post active treatment. Patient cooperation in wearing retainers is vital for maintaining the achieved results. ${ }^{[10]}$

Informed consent form: In today's era of dental practice, patients are the consumers while the doctors are the service care providers. Naturally, legal doctrine of informed consent is plays a pivotal role in everyday practice which has instilled fear in most dental health care professionals. According to the guidelines laid down by the American Association of Orthodontists, an informed consentought to include details such as treatment results and duration, presence and level of discomfort, risk of relapse, extractions and orthognathic surgery, possibilities of decalcification and dental caries, root resorption; periodontal diseases; risk of temporomandibular disorders, status of impacted and ankylosed teeth, allergies and usage of temporary anchorage devices in treatment. ${ }^{11}$ The orthodontist 
should attach documents of acknowledgement, a consent form and an authorization letter for the usage of patient information and their treatment records in case of future publication. ${ }^{[11]}$

Medicolegal Concerns: It is the medicolegal responsibility of the orthodontist to take radiographs and photographs of the patient before the start of the treatment, after getting a clear consent from them. These records are useful for screening any pathologies or anomalies, planning treatment mechanics for achieving best results, comparison of treatment results achieved and to get a second opinion if needed. Patients records must be filled and maintained in good condition even after orthodontic treatment. Using patients records, especially extraoral photographs for publication and research should be done only after patients' written approval. ${ }^{[11,12]}$

In case of referral of the patient to another orthodontist the copy of the patient's record must be saved. A detailed history if the patient, treatment plan and the progress of the treatment so far has to be transferred to the referral orthodontist. This must be done to ensure that no difficulties are faced both by the orthodontist and the patent in finishing the treatment. A clear and detailed consent should also be enclosed form both the parties.

Similarly, if the patient wants to discontinue the treatment due to personal reasons, orthodontist should explain the ill effects of incomplete treatment in case of extraction, and chances of relapse. Patients written consent should be obtained before removal of the appliance. ${ }^{[7,13]}$

Sterilization Protocols: Strict sterilization protocol must be meticulously followed must be strictly followed in the dental office. Clean sterile instruments, orthodontic attachments and wires should be used for each and every patient. The Operating orthodontist should wear personal protection equipments and maintain a sterile working environment. ${ }^{[14]}$

\section{Discussion}

Ethics is a subject of necessity which is considered in all aspects of life either consciously or unconsciously. ${ }^{1}$ Health care professionals possess training and expertise which the patients lack, making the treating orthodontist responsible for the decision making in regards to the treatment offered to the patient. ${ }^{[15]}$

It was suggested by Johnson in 1946 that a philosophical approach to ethics is in fact better than the dualistic approach which will allow the dental professional to pursue an ethical practice following a more eclectic approach. ${ }^{16}$ Ethical problems arise from conflicts between orthodontist's obligation to deliver best care to the patient and the need to respect their decisions. These are based on the underlying ethical principles of beneficence, and respect for autonomy of patients. ${ }^{[8]}$ Orthodontists should clearly outline the indicated treatment plan, the benefits and burden of the procedure as well as the consequence of no treatment. Information shared in an open fashion, should enable the patients and parents, in case of pediatric patients, to freely participate and make decisions.

Studies have shown that dental practitioners do not strictly follow an ethical clinical practice. ${ }^{[17-19]}$ Hence, regulatory bodies such as state dental councils and orthodontic societies should form ethical committees to actively monitor and guide the orthodontists to perform a healthy practice abiding by the codes of ethics. ${ }^{[12]}$

\section{Conclusion}

In the present scenario of dental practice,Ethics is of immense significance that gives an important dimension to patient care. Professionalism dictates profession as a correction to commercialism and is built on prioritizing professional ethics over personal interests or business.

The codes of ethics require an orthodontist to act in the best interest of patients regardless of their financial status. ${ }^{[8]}$ Abiding by these basic ethical codes will not only give simple moral justification in their duty as an orthodontist but also will protect them legally as a professional in providing the best healthcare to the community.

\section{Ethical Clearance: Nil}

Source of Funding: Meenakshi Academy of Higher Education and Research, Chennai, India

\section{Conflict of Interest: Nil}

\section{References}

1. Prasad M, Manjunath C, Krishnamurthy A, Shilpashree KB. Ethics in Dentistry-A Review.

2. Ozar DT. Dentistry.

3. Jonsen AR, Siegler M, Winslade WJ. Clinical ethics: a practical approach to ethical decisions in clinical medicine. 
4. Davison JA. Legal and Ethical Considerations for Dental Hygienists and Assistants.

5. Textbook of Preventive and Community Dentistry - 2nd Edition

6. Jerrold L. Ethical considerations regarding the timing of orthodontic treatment. American journal of orthodontics and dentofacial orthopedics. 1998 Jan 1;113(1):85-90.

7. Paranhos LR, Salazar M, Torres FC, Pereira AC, Silva RF, Ramos AL. Profile evaluation of orthodontics professionals as for their legal actions. Dental Press Journal of Orthodontics. 2011 Oct;16(5):127-34.

8. Mouradian WE, Lena Omnell M, Williams B. Ethics for orthodontists. The Angle Orthodontist. 1999 Aug;69(4):295-9.

9. Silva RH, Musse JD, Melani RF, Oliveira RN. Surgeon dentist's civil liability: the technical assistant's importance. Revista Dental Press de Ortodontia e Ortopedia Facial. 2009 Dec;14(6):6571.

10. Pratt MC, Kluemper GT, Lindstrom AF. Patient compliance with orthodontic retainers in the postretention phase. American Journal of Orthodontics and Dentofacial Orthopedics. 2011 Aug 1;140(2):196-201.

11. INFORMED CONSENT for the Orthodontic Patient Risks and Limitations of Orthodontic Treatment.

12. Devadiga A. What's the deal with dental records for practicing dentists? Importance in general and forensic dentistry. Journal of forensic dental sciences. 2014 Jan;6(1):9.

13. Paranhos LR, Benedicto ED, Fernandes MM, Queluz DD, Daruge E, Torres FC. Ethical and legal considerations on professional liability of the orthodontist. Dental Press Journal of Orthodontics. 2012 Dec;17(6):146-53.

14. Mithun K, Ashith MV, Harshitha V, Pereira VA, Kumari D. Infection control in orthodontics: A review. Indian Journal of Forensic Medicine \& Toxicology. 2018;12(2):10-5.

15. Mahfouz M, Mahfouz Y. Ethical Dilemmas in Orthodontics. Journal of Advances in Medicine and Medical Research. 2016 Jun 4:1-8.

16. Prasad DK, Hegde C, Jain A, Shetty M. Philosophy and principles of ethics: Its applications in dental practice. Journal of Education and Ethics in Dentistry. 2011 Jan 1;1(1):2.

17. Kesavan R, Mary AV, Priyanka M, Reashmi B. Knowledge of dental ethics and jurisprudence among dental practitioners in Chennai, India: A cross-sectional questionnaire study. Journal of Orofacial Sciences. 2016 Jul 1;8(2):128.

18. Janakiram C, Gardens SJ. Knowledge, attitudes and practices related to healthcare ethics among medical and dental postgraduate students in south India.

19. Milgrom P, Fiset L, Whitney C, Conrad D, Cullen T, O'Hara D. Malpractice claims during 1988-1992: a national survey of dentists. Journal of the American Dental Association (1939). 1994 Apr;125(4):4629. 